\title{
Evaluation of disaster nursing competencies for undergraduate nursing students in China: a study using a modified Delphi technique
}

Wang Yuan ${ }^{1 \#}$, Zhang Chao ${ }^{1 \#}, \mathrm{Xu} \mathrm{Ping}^{1}$, $\mathrm{Liu} \mathrm{Wei}^{2}$, Song Tianjiao ${ }^{1}$, Wang Taiwei ${ }^{1}$, Nie Wenbo ${ }^{1 *}$, Zhao Lijing ${ }^{*}$

1 School of Nursing, Jilin University, Changchun, China, 130021.

2 Jilin Academy of Environmental Science, Changchun, China, 130021.

$\#$, equally contributors

*, Addresses for correspondence and reprint requests:

Zhao Lijing, M.D., Ph.D. Department of Rahabilitaiton, School of Nursing, Jilin University, 965

Xinjiang Street, Changchun, China, 130021, Tel: +86 13756281155 Email: zhao_lj@jlu.edu.cn

Nie Wenbo, M.D., Tel: +86 13504336189, Email: Niewb@ljlu.edu.cn.

\begin{abstract}
This study aims to set up a system to evaluate nursing competencies in disaster preparedness for undergraduates and influencing factors in China. The evaluation system was established by using the modified Delphi. There were 3 phases in this study: 1) an environmental scan; 2) setting framework; 3) adjusting indicators based on the modified Delphi. The questionnaire for these undergraduates lied in their basic information and assessment of nursing competencies in disaster preparedness. Based on the survey, the average score of nursing competencies in disaster preparedness for undergraduate nursing students surveyed was 3.10 (out of 5). In addition, gender, grade, inhabitation, and disaster drills of the students were four factors affecting nursing competencies of undergraduates. This study indicates that the present nursing competencies in disaster preparedness for undergraduates are weak, and it is essential to strengthen nursing education in disaster preparedness for undergraduates.
\end{abstract}

Keyword: nursing student; disaster preparedness; China; the modified Delphi

\section{Introduction:}

With high frequency of large-scale, long-term and intensive disasters globally, disaster response and aid have been a focus. Disasters affect more than 255 million of people in the world, resulting in mortality of nearly 75,000 worldwide each year [1]. China has suffered high frequency and intensity of such disasters[2] as earthquakes, droughts, floods, typhoons, storms, coal mining disasters, traffic accidents, and terrorist attacks. These disasters led to chaos, mass casualties and property damage, brought out long-term social, physical, psychological, environmental, and economic consequences, endangered individual health, and overloaded the medical system [3]. Accompanied with inherent risks of high mortality and morbidity, these disasters are so complex as to be dealt with specific knowledge and skills. So indispensable well-trained are. [4]. As the largest group in the healthcare personnel, nurses play an increasingly important role in dealing with disasters [5]. When disasters occur, nurses act as in rescuers, care givers, educators, managers, and coordinators, and play an indispensable role in duration of disasters [6]. Nursing competencies of disaster preparedness affect directly consequences of rescuing. Nurses should be qualified for contingencies, professional activities skills, to provide adequate medical care before and after a disaster by participating in prevention, mitigation, preparation, and rescuing operations [7]. However, studies have shown that there is a gap between nurses' education and training, and requirements in emergencies yet. [3, 8]. Some nurses are not qualified adequately to deal with disasters in offering sufficient assistance [9].

As an important reserved force for medical staff, undergraduate nursing students are top in the list dealing with disasters. In peace times or in the pre-disaster phase, all 
nursing students should be urgent to acquire knowledge and skills related to medical care and disaster preparedness for unexpectations [10]. However, a review indicates that there were few studies on nursing competencies of undergraduates in disaster preparedness in China.

This study aimed to assess comprehensively medical care and disaster preparedness for undergraduate nursing students in China, and analyze the factors that influence competencies of undergraduates in dealing with disasters.

\section{Method}

\subsection{An investigation system established} 2.1.1Phase I

In this study an environmental scan of a literature review was conducted: 1) trends and issues on disaster nursing practice and education provincially, territorially, nationally, and internationally. 2) the criteria of disaster nursing competencies. A literature search was conducted in the databases of PubMed, The Cochrane Library, CINAHL, and NDNQI online, with search terms of nursing competencies in disasters, disaster preparedness, and nursing education.

\subsubsection{Phase II}

The research group were engaged in an iterative process of drawing up, reviewing, and revising nursing competencies in disaster preparedness, which were selected from the ICN Framework of Disaster Nursing Competencies, formulated jointly by the World Health Organization (WHO) and the International Council of Nurses (ICN) (2009) [11]. With reference to the definition of emergency preparedness competency in literature reviews [12], nurses are qualified with comprehensive knowledge, skills and performance to prepare and respond to disasters such as suspiciously chemical, biological, and radiological events.

\subsubsection{Phase III}

This phase consisted of two round surveys by using the modified Delphi. A total of 19 experts were participated in this study, with the inclusion criteria of: 1) teaching on acute or serious illnesses in key universities in China for more than 5 years, or working on acute or serious illnesses in hospitals (3-A typed hospitals) for more than 10 years, or engaging on health administration, nursing-related work in disasters; 2) rich professional theories or practical experience; 3) being ready to participate in consultation, promising to keep two rounds of research in this study; 4) being participated in disaster relief or engaged in related work on disaster nursing; 5)holding bachelor degree or above. These experts were communicated by e-mails or letters.

The first round was involved in: 1) instructions and requirements in developing questionnaire; 2) in the questionnaire for disaster nursing competency index, there were 3 first-class indicators, 11 second-class indicators, and 39 third-class indicators. Each item was rated into five categories: unimportant, less important, generally important, important, and very important according to the Likert scale. Categories are assigned 1 to 5 points respectively. Experts could add, delete or modify the items in the comments column, 3) experts' information. (gender, age, employment, years of administration, education, position, title, etc.), proficiency in disaster nursing and the 
judgment to this study.

The second round was involved in integrating of experts' opinions and statistical results. The questionnaire in the first round was revised, deleted and supplemented to form the second round survey involved in: 1) experts' feedback in the first round; 2) instructions and requirements of questionnaire filling; 3) the questionnaire with disaster preparedness nursing competencies index of 3 first-class indicators, 11 second-class indicators, and 39 third-class indicators. The evaluation criteria for each item were same as those in the first round, and the statistical results in the first round were attached. The experts judged the first round results with reference to the feedback from the first round, and proposed further amendments.

In this study, Delphi was the key method. 19 participants were experts majoring in disaster-nursing in universities, hospitals, national administrations of nursing ,and other health professionals attending the survey.

With data analyzed, the amendment was made: a three-class indicator modified, a three-class indicator added, and a third-class indicator deleted. The final questionnaire was made up of 3 first-class indicators, 11 second-class indicators, and 39 third-class indicators.

In two rounds of expert survey the positive coefficients were $100 \%$ and $94.7 \%$ respectively, the authority coefficient was 0.74 , and the coordination coefficient was 0.221-0.439. The difference was statistically significant by chi-square test $(P 1<0.05$, $P 2<0.01)$. This indicates that the experts' opinions are quite coherent and the indicators are reliable.

\subsection{The survey of undergraduates' nursing competencies in disaster preparedness}

In this study, 426 undergraduates were selected from 6 universities in 4 provinces of Jilin, Chongqing, Hebei and Gansu representing Northern China, Southern China, Eastern China, and Western China in turn. The undergraduates were 5 grades students who had no actual experience in disaster nursing.

The Informed Consent Forms were obtained from these students prior to inclusion. They were asked to fill out the questionnaire including basic information and disaster nursing competencies questionnaire. And it consisted of a self-report rated scale using a 5-point Likert scale with 1 representing "poor mastery", and 5 representing "mastered very well". The students' disaster nursing competencies were measured and classified into 4 levels in terms of low, medium, high and very high.

Two groups were selected randomly from 426 undergraduates for pre-experiment ahead of two weeks. The Cronbach's a coefficient was 0.971 and the CVI was 0.906 .

\subsection{Data analysis}

2.4

Data were analyzed by SPSS of version19.0. The results were presented in descriptive (frequency, percentage, and mean) and inferential statistics (analysis of variance, t-test, and Pearson correlation). Statistical significance was defined as $p<0.05$.

\section{Results}

\subsection{The demographic characteristics of undergraduates}

All participants were undergraduate nursing students, and most of whom were female 
(81.22\%). $59.39 \%$ of them came from towns, and $73.47 \%$ had been participated in organized emergency disaster drills. $25.35 \%$ had the experience in disasters. $29.11 \%$ had not heard of or made sense of disasters nursing (Table1).

Table 1. The demographic characteristics of the students.

\begin{tabular}{lll}
\hline Variable & Number & Percent \\
\hline
\end{tabular}

\section{Gender}

Male

80

$18.78 \%$

Female

346

$81.22 \%$

Grade

Grade one

87

$20.42 \%$

Grade two

88

$20.66 \%$

Grade three

151

$35.45 \%$

Grade four

62

$14.55 \%$

Grade five

38

$8.92 \%$

Hometown

Rural area

173

$40.61 \%$

Urban area

253

$59.39 \%$

\section{Whether participated emergency disaster drills}

Yes

313

$73.47 \%$

No

113

$26.53 \%$

\section{Experience in disasters Yes/No}

Yes

108

$25.35 \%$

No

318

$74.65 \%$

No sense of disaster nursing

124

$29.11 \%$ 


\subsection{Nursing competencies of undergraduates in disaster preparedness}

Overall disaster preparedness nursing competency score of nursing students was 3.10 points (the total score of 5 points), which was on the edge of passing. The scores for the second-class indicators were: knowledge system score of 2.58 points, practical skill score of 3.07 points, and physical and mental diathesis score of 3.33 points. (the total score of 5 points).

According to the overall scores, the disaster preparedness nursing competencies was divided into 4 levels, 1) very high: $4.46 \%$ of participants scored more than 4 points; 2 ) high: $34.74 \%$ scored 3-4 points; 3)medium: $34.74 \%$ of scored 2-3 points; 4)low: $26.06 \%$ scored less than 2 points (Table2).

Table 2. The students' disaster nursing competencies.

\begin{tabular}{llll}
\hline Level & Score & Number & Percentage \\
\hline Very high & $4-5$ & 19 & $4.46 \%$ \\
High & $3-4$ & 148 & $34.74 \%$ \\
Medium & $2-3$ & 148 & $34.74 \%$ \\
Low & $1-2$ & 111 & $26.06 \%$ \\
Average / Total & 3.1 & 426 & $100.00 \%$ \\
\hline
\end{tabular}

According to the data, the overall nursing competencies of disaster preparedness for the nursing students was weak, and high-scored students were few and far between. When disasters break our suddenly, it is difficult for nursing students to play an important role as the backup nursing staff.

\subsection{The link between the student's disaster preparedness nursing competencies and the demographic data}

According to the data analysis, the overall score, practical skills and physical and mental quality of nursing students were related obviously to gender $(P<0.01)$, with male nursing students' scores being higher than female ones. Furthermore, disaster nursing competencies was positively correlated with grades $(P<0.01)$, with the scores of high-grade students being higher than those in low grades. Nursing students from rural areas scored higher than urban nursing students $(P<0.01)$. Whether they had been participated in emergency disaster drills or not was another important factor affecting nursing competencies of nursing students $(P<0.01)$, with those who had been participated in emergency disaster drills scored higher than those who hadn't (Table 3).

According to the univariate analysis of variance (ANOVA), no significant links statistically were found between 'whether they were the only-child in a family', 'whether they experienced disasters', in disaster preparedness nursing competencies. $(P>0.05)$. 
Table 3. The factors on students' disaster nursing competencies.

\begin{tabular}{|c|c|c|c|c|c|}
\hline \multirow[t]{2}{*}{ Factor } & \multirow[t]{2}{*}{ Number } & \multicolumn{3}{|c|}{ Score } & \multirow[b]{2}{*}{ Total } \\
\hline & & $\begin{array}{l}\text { Knowledge } \\
\text { system }\end{array}$ & $\begin{array}{l}\text { Practical } \\
\text { skills }\end{array}$ & $\begin{array}{l}\text { Physical and mental } \\
\text { diathesis }\end{array}$ & \\
\hline \multicolumn{6}{|l|}{ Gender } \\
\hline Male & 80 & 2.5 & 3.11 & 3.5 & 3.19 \\
\hline Female & 346 & 2.6 & 3.06 & 3.3 & 3.07 \\
\hline$P$ value & & $>0.05$ & 0.01 & 0.01 & 0.01 \\
\hline \multicolumn{6}{|l|}{ Grade } \\
\hline Grade one & 87 & 1.57 & 2.26 & 2.8 & 2.21 \\
\hline Grade two & 88 & 2.21 & 2.3 & 2.93 & 2.48 \\
\hline Grade three & 151 & 2.46 & 2.96 & 3.16 & 2.86 \\
\hline Grade four & 62 & 2.85 & 3.41 & 4.16 & 3.47 \\
\hline Grade five & 38 & 3.25 & 4 & 4.2 & 3.82 \\
\hline$P$ value & & 0.01 & 0.01 & 0.01 & 0.01 \\
\hline \multicolumn{6}{|l|}{ Hometown } \\
\hline Rural area & 173 & 2.8 & 3.3 & 3.5 & 3.2 \\
\hline Urban area & 253 & 2.43 & 2.91 & 3.22 & 3.03 \\
\hline$P$ value & & 0.01 & 0.01 & 0.01 & 0.01 \\
\hline \multicolumn{6}{|c|}{$\begin{array}{l}\text { Whether } \\
\text { participated } \\
\text { emergency disaster } \\
\text { drills }\end{array}$} \\
\hline Yes & 313 & 2.65 & 3.11 & 3.47 & 3.2 \\
\hline No & 113 & 2.39 & 2.96 & 2.94 & 2.82 \\
\hline$P$ value & & 0.01 & 0.01 & 0.01 & 0.01 \\
\hline Total & 426 & 2.58 & 3.07 & 3.33 & \\
\hline
\end{tabular}

\section{Discussion}

4.1 The nursing competencies of disaster preparedness for undergraduate nursing students

This study shows that nursing competencies of disaster preparedness for undergraduate nursing students are: the physical and mental diathesis of 3.33, the 
practical skills of 3.07, and knowledge system of 2.58. The total score of 3.10 in nursing competencies of disaster preparedness means that nursing students are weak in nursing competencies of disaster preparedness, which is consistent with previous research results [13-16]. These findings also makes it confirmed that nurses are unprepared for disaster work $[9,17]$.

In addition, many studies have exposed that China's disaster nursing education and training is less effective [18, 19]. Alim et al. emphasized that disaster preparedness education is essential for nurses to be qualified to respond to any disasters [20]. Poor nursing competencies of disaster preparedness for nursing students weaken directly disaster nursing care for nursing staff. Although disaster nursing education for undergraduates is different from that for professional nursing staff, nursing students have to be qualified to cope with disasters when they graduate [21]. Disaster preparedness is required in the undergraduate nursing curriculum [22]. Incorporating disaster care into undergraduate nursing programs not only strengthens the ability of health workers to respond, but also provides graduates with a foundation to enter the workforce and develop further through on-job training and continuous training [3].

Ireland et al. (2006) advised advanced nursing students to acquire sufficient skills and experience and strengthen basic disaster management training so as to provide valuable assistance in the events of mass casualties [23]. As a reserved force for nursing staff, nursing students should take disaster nursing knowledge and skills training courses or seminars in school, which not only improve their disaster nursing competencies, but also increase the overall nursing staff's proficiency of disaster nursing preparation.

\subsection{The link between undergraduates' disaster preparedness nursing competencies and related factors}

4.2.1 Gender has some impact on disaster nursing competencies

It was shown that male nursing students scored more prominently than females in overall score, practical skills and knowledge system. Similarly, Tan et al. found that male college students had higher preparedness than females [24]. Male nurses have more mental and physical advantages in working and are receptive to the latest scientific knowledge, which helps them improve nursing competencies.

\subsubsection{Grades has some impact on disaster preparedness nursing competencies}

It was shown that undergraduates' disaster preparedness nursing competencies were positively correlated with grades. The higher the grade, the higher the score of the disaster preparedness nursing competencies, among which freshmen scored the lowest, below the average, and the students in graduating grade scored the highest. Disaster care preparedness of the students in higher grades were better than that of lower grades [15]. It might be related to the courses carried out by universities. In the beginning, freshmen take foundation courses. Later, professional courses are gradually added, and the clinical internship of graduation year plays a more important role on the practical skills.

\subsubsection{Inhabitation has some impact on disaster preparedness nursing competencies}

It was shown that the nursing students from rural areas got higher scores in all aspects than urban nursing students. This result was conflicted with one of previous findings 
that social situations are related with college students' competencies in disaster preparedness, and urban students has better preparedness than rural students [24].

The reason why rural students scored higher than urban students in disaster preparedness nursing ability is not understood fully. This may be explained by their families and parenting styles. Compared with urban nursing students, rural nursing students had a stronger ability to live and practice independently

-Emergency disaster drills have some impact on disaster preparedness nursing competencies

It was showed that nursing students who had participated in emergency disaster drills scored higher than those who did not. Training and drills can improve disaster preparedness and ability of undergraduates [25].

\section{Limitations of research}

4.3 In this study only nursing students were recruited from Jilin, Chongqing, Hebei and Gansu rather than other provinces due to limited time and manpower. Therefore, this study is not applicable to nursing populations throughout China. Larger and more samples should be recruited to future research.

\section{Conclusion}

This study established an evaluation system for disaster preparedness nursing competencies of undergraduate nursing students, and disaster preparedness nursing competencies were evaluated. It was showed that due to insufficient corresponding courses and drills, undergraduate nursing students' competencies of disaster preparedness nursing were deficient, so it is necessary to set relevant courses or drills to improve nursing competencies and the overall level of nursing staff of disaster-relief.

Author Contributions: Conceptualization, Wang Yuan, Zhang Chao and Zhao Lijing ; ;methodology, Wang Yuan, Zhang Chao, investigation, Zhang Chao, Xu Ping, data curation, Wang Yuan, Xu Ping, writing — original draft preparation, Wang Yuan, $\mathrm{Xu}$ Ping and Wang Taiwei, writing - reviewing and editing, Wang Yuan, Song Tianjiao and Nie Wenbo, supervision, Liu Wei, Zhao Lijing. All authors have read and agreed to the published version of the manuscript.

Funding: This research received no external funding.

Conflicts of Interest: The authors declare no conflict of interest. 


\section{References}

1. Zarea, K., et al., Disaster nursing in Iran: Challenges and opportunities. Australasian emergency nursing journal, 2014. 17(4): p. 190-196.

2. Yan, Y., et al., Disaster nursing skills, knowledge and attitudes required in earthquake relief: Implications for nursing education. International nursing review, 2015. 62(3): p. 351-359.

3. Achora, S. and J.K. Kamanyire, Disaster Preparedness: Need for inclusion in undergraduate nursing education. Sultan Qaboos University Medical Journal, 2016. 16(1): p. e15.

4. Siemon, M., J. Hackwith, and K. Monson, Development of a Disaster Nursing Competencies Assessment Instrument for Baccalaureate Nursing Education. Journal of Nursing Education, 2019. 58(4): p. 229-233.

5. Loke, A. and O. Fung, Nurses' competencies in disaster nursing: Implications for curriculum development and public health. International journal of environmental research and public health, 2014. 11(3): p. 3289-3303.

6. Zhang, Y.Y., et al., Disaster nursing development in China and other countries: a bibliometric study. Journal of Nursing Scholarship, 2018. 50(5): p. 567-576.

7. Fung, O.W., A.Y. Loke, and C.K. Lai, Disaster preparedness among Hong Kong nurses. J Adv Nurs, 2008. 62(6): p. 698-703.

8. Chan, S.S., et al., Development and evaluation of an undergraduate training course for developing International Council of Nurses disaster nursing competencies in China. J Nurs Scholarsh, 2010. 42(4): p. 405-13.

9. Littleton-Kearney, M.T. and L.A. Slepski, Directions for disaster nursing education in the United States. Critical care nursing clinics of North America, 2008. 20(1): p. 103-109.

10. Fung, W.M., K.Y. Lai, and A.Y. Loke, Nurses' perception of disaster: implications for disaster nursing curriculum. J Clin Nurs, 2009. 18(22): p. 3165-71.

11. Organization, W.H., International Council of Nurses. ICN framework of disaster nursing competencies. 2009. 2015.

12. Slepski, L.A., Emergency preparedness: Concept development for nursing practice. Nursing Clinics, 2005. 40(3): p. 419-430.

13. Bayraktar, N. and M. Yildirim, Senior Undergraduate Nursing Students' Perceptions of Disaster Preparedness: A Descriptive Study. Disaster Med Public Health Prep, 2016: p. $1-5$.

14. Choe, M.A., et al., Japanese and Korean Nursing Students' Motivation for Joining Disaster Relief Activities as Nurses in the Future. J Trauma Nurs, 2017. 24(3): p. 208-218.

15. Öztekin, S.D., et al., Undergraduate nursing students' perceptions about disaster preparedness and response in I stanbul, T urkey, and M iyazaki, J apan: A cross-sectional study. Japan Journal of Nursing Science, 2015. 12(2): p. 145-153.

16. !!! INVALID CITATION !!! .

17. Chen, T.F., et al., Construct validity and reliability of the Chinese version of the Disaster Preparedness Evaluation Tool in Taiwan. J Clin Nurs, 2015. 24(7-8): p. 1132-43.

18. Li, Y.H., et al., Disaster nursing experiences of Chinese nurses responding to the Sichuan Ya'an earthquake. Int Nurs Rev, 2017. 64(2): p. 309-317.

19. Wenji, Z., et al., Chinese nurses' relief experiences following two earthquakes: implications for disaster education and policy development. Nurse Educ Pract, 2015. 
15(1): p. 75-81.

20. Alim, S., M. Kawabata, and M. Nakazawa, Evaluation of disaster preparedness training and disaster drill for nursing students. Nurse Educ Today, 2015. 35(1): p. 25-31.

21. Adams, L.M., S.B. Canclini, and P.J. Frable, "Skip the infection, get the injection": a case study in emergency preparedness education. Nurse Educ Pract, 2015. 15(1): p. 58-62.

22. Jose, M.M. and C. Dufrene, Educational competencies and technologies for disaster preparedness in undergraduate nursing education: an integrative review. Nurse Education Today, 2014. 34(4): p. 543-551.

23. Ireland, M., et al., Integrating disaster preparedness into a community health nursing course: one school's experience. Disaster Manag Response, 2006. 4(3): p. 72-6.

24. Tan, Y., et al., Disaster Preparedness Among University Students in Guangzhou, China: Assessment of Status and Demand for Disaster Education. Disaster Med Public Health Prep, 2017. 11(3): p. 310-317.

25. Alim, S., M. Kawabata, and M. Nakazawa, Evaluation of disaster preparedness training and disaster drill for nursing students. Nurse education today, 2015. 35(1): p. 25-31. 